\title{
Discrete-Time Positive Periodic Systems with State and Control Constraints
}

\author{
Mustapha Ait Rami and Diego Napp
}

\begin{abstract}
Resumo-The aim of this paper is to provide an efficient control design technique for discrete-time positive periodic systems. In particular, stability, positivity and periodic invariance of such systems are studied. Moreover, the concept of periodic invariance with respect to a collection of boxes is introduced and investigated with connection to stability. It is shown how such concept can be used for deriving a stabilizing state-feedback control that maintains the positivity of the closed-loop system and respects states and control signals constraints. In addition, all the proposed results can be efficiently solved in terms of linear programming.
\end{abstract}

\section{INTRODUCTION}

The purpose of this paper is to study a special class of switched systems, namely discrete-time positive periodic systems that are subject to periodic switching. Historical roots of periodic systems can be traced back to the early works of Floquet [29] and Lyapunov [32] respectively in 1883 and 1896. There has been an increasing interest in such systems for which many flourishing results have been reported during the last three decades. The well established analysis and synthesis framework for LTI systems has been extended to this particular class of switched systems. Monographs [9] and [14] provide a wide scope on existing results. We mention, among others, a fundamental stability result in [15] using a periodic Lyapunov equation. Later, a periodic Riccati equation has been introduced and studied in [6], [7]. Stabilization techniques based on periodic Lyapunov and Riccati equations have been considered in [25], [40], [41]. Robustness and dissipativity issues have also been addressed, see for instance, [26], [34] and [39]. Important works about techniques that transform a discrete-time periodic system into a time-invariant one can be found in [9], [28], [31], [37], [38]. Surprisingly, few results exist that extend the framework of LTI positive systems to positive periodic systems. By definition, a positive periodic system keeps invariant the positive orthant, that is, its trajectory evolves in the positive orthant whenever it starts from it. For general references on positive systems one can consult [27], [30], [33]. Reachability, controllability, realizability and other fundamental properties of the peculiar class of positive periodic systems have been investigated in [17]-[22], [36]. However, the derived results for such systems are still modest in comparison to general periodic systems for which there exists an extensive literature. Positivity conditions for such systems under a periodic feedback have been studied in [24]. Agriculture applications of positive periodic systems can be found in [19], [22]. In [23] an extension to the class of descriptor positive periodic systems has been considered.

Mustapha Ait Rami is with LTI Dept., University of Picardie Jules Verne, France and Diego Napp is with Department of Mathematics, University of Aveiro, Portugal.

Corresponding author: diegonapp@gmail.com
On the one hand, this paper shows the intrinsic interplay between positivity, stability and periodic invariance of a discrete positive periodic system. On the other hand, the stabilization issue for such systems with states and control constraints is addressed. This paper completes previous stability and stabilization results in [11] since our conditions are necessary and sufficient. In addition, we study periodic-invariance concept for discrete positive periodic systems and establish a connection between their stability and periodic invariance with respect to a collection of boxes. Specifically, we show that a positive $T$-periodic system is asymptotically stable if and only if it has a periodically invariant and contractive collection of $T$ boxes. Based on this relationship we show how one can tackle the synthesis problem with respect to states and control component-wise constraints. Moreover, it is shown how to compute a larger inner estimate box of the region of attraction by using an adequate LP formulation. Indeed, LMI approach that has been used in [12], cannot handle state and control component-wise constraints. The proposed LP approach seems to be suitable in other contexts of control and estimation of LTI positive systems [1]-[5], [16], [35].

The reminder of the paper is organized as follows. The second section shows the interconnection between stability and periodic-invariance. In Section 3, an efficient numerical treatment is provided for stabilization. Section 4 considers the synthesis problem with constrained states and controls. In Section 5, it is shown how one can enlarge the domain of attraction based on LP optimization. Finally, Section 6 gives some concluding remarks.

Notation. $\mathbb{Z}_{+}$is the set of nonnegative integer numbers. $\mathbb{R}_{+}^{n}$ denotes the nonnegative orthant of the $n$-dimensional real space $\mathbb{R}^{n} . \mathbb{1}_{n}$ denotes the vector with all unitary entries $\left[\begin{array}{llll}1 & 1 & \cdots & 1\end{array}\right]^{T} \in \mathbb{R}^{n}$. For a real matrix (or a vector) $M, M>0$ means that its components are positive and $M \geq 0$ means that its components are nonnegative. Let $M_{1}, \ldots, M_{p}$ be square matrices, then $\operatorname{diag}\left(M_{1}, \ldots, M_{p}\right)$ is the block diagonal matrix whose $i$ th block is $M_{i}$. Also, for a vector $v, \operatorname{diag}(v)$ denotes the diagonal matrix whose $i$ th diagonal entry is $v(i) . \prod_{i=k}^{l} M_{i}$ denotes the matrix product $M_{l} \cdots M_{k}$ in the decreasing sense for integers $l$ and $k$ such that $l>k$. $I$ stands for the identity matrix of appropriate dimension.

\section{Stability And Periodic InVARiance}

The purpose of this section is to show the interplay between stability and periodic invariance for the following discrete-time T-periodic system

$$
x(t+1)=A(t) x(t),
$$


where $A(t) \in \mathbb{R}^{n \times n}, t \in \mathbb{Z}_{+}$. The matrix $A(t)$ is supposed to be periodic, that is for all $t \in \mathbb{Z}_{+}, A(t+T)=A(t)$ with $T \in \mathbb{Z}_{+}$its period.

It is well-known that system (1) is asymptotically stable, i.e., $\mathrm{x}(\mathrm{t})$ goes to zero when $t$ goes to infinity if and only if its monodromy matrix $A(T-1) \cdots A(1) A(0)$ is Schur, i.e. its eigenvalues have modulus less than one. The following result provides other equivalent conditions for stability of system (1), see for instance [8], [9], [14].

Lemma 2.1: For a discrete-time T-periodic system the following statements are equivalent.

(i) System (1) is asymptotically stable.

(ii) $A(T-1) \cdots A(1) A(0)$ is Schur.

(iii)

$$
\mathcal{A}_{T}:=\left(\begin{array}{cc}
0 & A(0) \\
\operatorname{diag}(A(1), \ldots, A(T-1)) & 0
\end{array}\right)
$$

is Schur.

iv) There exists a cyclic permutation $\sigma$ of $\{0, \ldots, T-1\}$ such that

$$
A(\sigma(T-1)) A(\sigma(T-2)) \ldots A(\sigma(0)) \text { is Schur. }
$$

v) All cyclic permutations of $\{A(0), A(1), \cdots, A(T-1)\}$ are Schur, that is

$$
\rho(A(i) A(i+1) \cdots A(i+T-1))<1,0 \leq i \leq T-1 .
$$

The matrix $\mathcal{A}_{T}$ defined previously in Lemma 2.1 characterizes a shift-invariant representation for a periodic linear discrete-time system. Other lifting techniques have been considered extensively in the literature, see for instance [9], [28], [31], [38].

In what follows we define the positivity notion.

Definition 2.2: System (1) is said to be positive if for any nonnegative initial condition $x\left(t_{0}\right)$ at any initial time $t_{0} \geq 0$ the corresponding trajectory remains in the positive orthant.

Remark 2.3: Indeed, if system (1) is positive for any nonnegative initial condition $x\left(t_{0}\right)$ at any initial time $t_{0} \geq 0$, then the modes of system (1) are necessarily nonnegative: $A(i) \geq 0$ for all $i$. This is also a trivial sufficient condition for positivity.

Throughout this paper we make extensive use of the following stability result for discrete positive systems, see for instance [27].

Lemma 2.4: The system $z(t+1)=M z(t)$ with $M \geq 0$ is asymptotically stable if and only if there exists a vector $\lambda>0$ such that $M \lambda<\lambda$.

Remark 2.5: From the previous Lemma we can deduce that if the monodromy matrix is positive then T-periodic system (1) is asymptotically stable if and only if there exists $\lambda>0$ such that $A(T-1) \cdots A(1) A(0) \lambda<\lambda$. Note that the following stability condition reported in [11] is only sufficient:

There exist vectors $\lambda_{0}>0, \ldots, \lambda_{T-1}>0$ such that

$\lambda_{0}-A(T-1) \lambda_{T-1}>0$, and $A(i) \lambda_{i}=\lambda_{i+1}, i=0, \ldots, T-2$.

This fact can be shown by the following counterexample with the following modes $A(0)=\left(\begin{array}{cc}0.5 & 1 \\ 0 & 0\end{array}\right)$ and $A(1)=$ $\left(\begin{array}{ll}1 & 1 \\ 0 & 1\end{array}\right)$. The associated periodic system is stable since its monodromy matrix $A(1) A(0)=\left(\begin{array}{cc}0.5 & 1 \\ 0 & 0\end{array}\right)$ has 0 and 0.5 as eigenvalues. However, the equality $A(0) \lambda_{0}=\lambda_{1}$ in condition (3) can never be satisfied for any $\lambda_{1}>0$, since the second component of $A(0) \lambda_{0}$ is always zero. Indeed, the true necessary and sufficient condition involves $\lambda_{1} \geq 0, \ldots, \lambda_{T-1} \geq 0$ with possibly zeros components and reads:

There exist $\lambda_{0}>0$ and $\lambda_{1} \geq 0, \ldots, \lambda_{T-1} \geq 0$ such that

$\lambda_{0}-A(T-1) \lambda_{T-1}>0$ and $A(i) \lambda_{i}=\lambda_{i+1}, i=0, \ldots, T-2$.

As a matter of fact the above condition is equivalent to the existence of $\lambda>0$ such that $A(T-1) \cdots A(1) A(0) \lambda<\lambda$. This can be easily seen by defining $\lambda_{0}=\lambda$ and $\lambda_{i}=$ $\prod_{j=0}^{i-1} A(j) \lambda$ for $i=1, \ldots, T-1$. Hence, the stability condition given by (4) is necessary and sufficient. Unfortunately, due to technical reasons condition (4) cannot be used for the synthesis problem because it involves $\lambda_{i}$ that are not all strictly positive. Later, we shall provide necessary and sufficient conditions for stability synthesis that are easily checkable.

Next, we define the periodic invariance concept with respect to a collection of $\mathrm{T}$ sets. We shall introduce a slightly different definition of the concept of periodically invariant set introduced in [10]. Further, we shall show that our extended concept of invariance is naturally and inherently connected to the stability of system (1).

Definition 2.6: A collection of T sets $\mathcal{S}_{0}, \mathcal{S}_{1}, \ldots, \mathcal{S}_{T-1}$ is called periodically invariant for system (1) if $x(0) \in \mathcal{S}_{0}$ implies that for all $N \in \mathbb{Z}_{+}, x(i+N T) \in \mathcal{S}_{i}$ for $i=0, \ldots, T-1$.

For a set $\mathcal{S}$, define by $\dot{\mathcal{S}}$ its interior and by $\overline{\mathcal{S}}$ its closure. Then a collection of T sets $\mathcal{S}_{0}, \mathcal{S}_{1}, \ldots, \mathcal{S}_{T-1}$ is called periodically invariant and contractive for system (1) if $x(0) \in \overline{\mathcal{S}}_{0}$ implies that for all $N \in \mathbb{Z}_{+}, x(i+N T) \in \dot{\mathcal{S}}_{i}$ for $i=0, \ldots, T-1$.

For a given vector $v>0$, define an open box as $\mathbb{B}(v):=$ $\left\{x \in \mathbb{R}_{+}^{n} \mid x<v\right\}$ and note that its closure is given by $\overline{\mathbb{B}}(v):=\left\{x \in \mathbb{R}_{+}^{n} \mid x \leq v\right\}$. With connection to such box, the following result provides necessary and sufficient conditions for a collection of boxes to be periodically invariant and contractive for system (1).

Theorem 2.7: Let $\bar{x}_{0}>0, \bar{x}_{1}>0, \ldots, \bar{x}_{T-1}>0$ be given. Assume that system (1) is positive. Then, for any arbitrary initial condition of system (1) such that $x(0) \in \overline{\mathbb{B}}\left(\bar{x}_{0}\right)$, we have that its resulting trajectory satisfies $x(N T) \in \mathbb{B}\left(\bar{x}_{0}\right), x(1+$ $N T) \in \mathbb{B}\left(\bar{x}_{1}\right), \ldots, x(T-1+N T) \in \mathbb{B}\left(\bar{x}_{T-1}\right), \forall N \in \mathbb{Z}_{+}$. if and only if the following conditions hold

$$
\left\{\begin{array}{l}
A(0) \bar{x}_{0}<\bar{x}_{1} \\
A(1) A(0) \bar{x}_{0}<\bar{x}_{2} \\
\vdots \\
A(T-1) \cdots A(1) A(0) \bar{x}_{0}<\bar{x}_{0}
\end{array}\right.
$$

Proof: Necessity: Take $x(0)=\bar{x}_{0}$. Since $x(1) \in \mathbb{B}\left(\bar{x}_{1}\right)$ then $x(1)<\bar{x}_{1}$ and therefore we have $x(1)=A(0) x(0)=$ $A(0) \bar{x}_{0}<\bar{x}_{1}$. Further, by positivity assumption on the system the inequality $x(2)<\bar{x}_{2}$ implies that $x(2)=A(1) A(0) x(0)=$ $A(1) A(0) \bar{x}_{0}<\bar{x}_{2}$. Same reasoning proves that the rest of the inequalities given by conditions (5) holds true. 
Sufficiency: Let $x(0)$ be any initial condition such that $0 \leq$

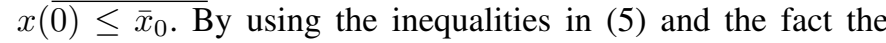
modes of system (1) are positive, we have that the trajectory corresponding to $x(0)$ satisfies for $r=0, \ldots, T-1$

$$
\begin{aligned}
x(r+N T) & =\left(\prod_{j=0}^{r} A(j)\right)\left(\prod_{j=0}^{T-1} A(j)\right)^{N} x(0) \\
& \leq\left(\prod_{j=0}^{r} A(j)\right)\left(\prod_{j=0}^{T-1} A(j)\right)^{N} \bar{x}_{0} \\
& \leq \prod_{j=0}^{r} A(j) \bar{x}_{0}<\bar{x}_{r},
\end{aligned}
$$

i.e., $x(r+N T) \in \mathbb{B}\left(\bar{x}_{r}\right)$.

More important is the following result which characterizes asymptotic stability in terms of periodic invariance and contractivity.

Theorem 2.8: A positive periodic system is asymptotically stable if and only if it has a periodically invariant and contractive collection of boxes associated to $\bar{x}_{0}>0, \bar{x}_{1}>$ $0, \ldots, \bar{x}_{T-1}>0$, that is, if $x(0) \in \overline{\mathbb{B}}\left(\bar{x}_{0}\right)$, the resulting trajectory satisfies $x(N T) \in \mathbb{B}\left(\bar{x}_{0}\right), x(1+N T) \in \mathbb{B}\left(\bar{x}_{1}\right), \ldots, x(T-$ $1+N T) \in \mathbb{B}\left(\bar{x}_{T-1}\right), \forall N \in \mathbb{Z}_{+}$

Proof: Assume that a positive periodic system has a periodically invariant and contractive collection of boxes $\mathbb{B}\left(\bar{x}_{0}\right), \ldots, \mathbb{B}\left(\bar{x}_{T-1}\right)$. Since $x(T) \in \mathbb{B}\left(\bar{x}_{0}\right)$ we have that $A(T-1) \cdots A(1) A(0) \bar{x}_{0}<\bar{x}_{0}$. Also, by positivity assumption we have $A(T-1) \cdots A(1) A(0) \geq 0$ which together with $A(T-1) \cdots A(1) A(0) \bar{x}_{0}<\bar{x}_{0}$ guarantee stability by means of Lemma 2.1 and Lemma 2.4.

Conversely, consider any positive $\bar{x}_{0}$ such that $A(T-$ 1) $\cdots A(1) A(0) \bar{x}_{0}<\bar{x}_{0}$ which is guaranteed by positivity and stability of a T-periodic system. Note that it is possible to select $\bar{x}_{1}>0, \ldots, \bar{x}_{T-1}>0$ such that $A(0) \bar{x}_{0}<\bar{x}_{1}, A(1) A(0) \bar{x}_{0}<$ $\bar{x}_{2}, \ldots, A(T-2) \cdots A(0) \bar{x}_{0}<\bar{x}_{T-1}$. Thus, from the invariance and contractivity result given by Theorem 2.7, we can deduce that if $x(0) \in \overline{\mathbb{B}}\left(\bar{x}_{0}\right)$, the resulting trajectory satisfies $x(N T) \in \mathbb{B}\left(\bar{x}_{0}\right), x(1+N T) \in \mathbb{B}\left(\bar{x}_{1}\right), \ldots, x(T-1+N T) \in$ $\mathbb{B}\left(\bar{x}_{T-1}\right), \forall N \in \mathbb{Z}_{+}$.

\section{STABILIZATION}

In this section, we address stability synthesis with positivity constraint for the following periodic system given by

$$
\dot{x}(t)=A(t) x(t)+B(t) u(t)
$$

with $A(t) \in \mathbb{R}^{n \times n}, B(t) \in \mathbb{R}^{n \times p}$, such that $A(t+T)=A(t)$ and $B(t+T)=B(t)$ for all $t \in \mathbb{Z}_{+}$.

Here, the main problem under investigation is to find a periodic state-feedback $u(t)=K(t) x(t)$ such that the resulting closed-loop system $x(t+1)=[A(t)+B(t) K(t)] x(t)$ is positive and asymptotically stable.

In the rest of the paper, the two conditions given by the following linear inequalities will play a fundamental role in our development:

There exist $Y_{0}, \ldots, Y_{T-1} \in \mathbb{R}^{p \times n}$ and $\lambda_{0}, \ldots, \lambda_{T-1} \in \mathbb{R}^{n}$ satisfying

$$
A(i) \operatorname{diag}\left(\lambda_{i}\right)+B(i) Y_{i} \geq 0 \text { for } i=0,1, \ldots, T-1,
$$

$$
\left\{\begin{aligned}
\lambda_{0}>0, \lambda_{1}>0, \ldots, \lambda_{T-2}>0, \lambda_{T-1} & >0 \\
A(0) \lambda_{0}+B(0) Y_{0} \mathbb{1}_{n} & <\lambda_{1} \\
A(1) \lambda_{1}+B(1) Y_{1} \mathbb{1}_{n} & <\lambda_{2} \\
\vdots & \\
& \\
& \\
A(T-2) \lambda_{T-2}+B(T-2) Y_{T-2} \mathbb{1}_{n} & <\lambda_{T-1} \\
A(T-1) \lambda_{T-1}+B(T-1) Y_{T-1} \mathbb{1}_{n} & <\lambda_{0} .
\end{aligned}\right.
$$

The following result shows how one can look for a periodic gain $K(t)$ such that the closed-loop system is positive and asymptotially stable.

Theorem 3.1: There exists a stabilizing state-feedback law $u(t)=K(t) x(t)$ for system (6) with a periodic gain $K(t+$ $T)=K(t)$ that maintains the positivity of the closed-loop system if and only if there exist $Y_{0}, \ldots, Y_{T-1} \in \mathbb{R}^{p \times n}$ and $\lambda_{0}, \ldots, \lambda_{T-1} \in \mathbb{R}^{n}$ such that the linear inequalities (7) and (8) are feasible. Moreover, the resulting closed-loop system is positive and asymptotically stable under the control law $u(t)=K(t) x(t)$ such that

$$
K(t+N T)=Y_{t} \operatorname{diag}\left(\lambda_{t}\right)^{-1}, t \in\{0, \ldots, T-1\}, N \in \mathbb{Z}_{+} .
$$

Proof: Let us show the sufficiency of the proposed condition. Define $K(i)=Y_{i} \operatorname{diag}\left(\lambda_{i}\right)^{-1}$ for $i \in\{0, \ldots, T-1\}$. Since $\lambda_{0}, \ldots, \lambda_{T-1}$ are positive it holds that $A(i) \operatorname{diag}\left(\lambda_{i}\right)+$ $B(i) Y_{i} \geq 0$ if and only if

$$
\left[A(i) \operatorname{diag}\left(\lambda_{i}\right)+B(i) Y_{i}\right] \operatorname{diag}\left(\lambda_{i}\right)^{-1}=A(i)+B(i) K(i) \geq 0
$$

which proves the equivalence between the positivity of the closed-loop system and condition (7).

As $Y_{i}=K(i) \operatorname{diag}\left(\lambda_{i}\right), Y_{i} \mathbb{1}_{n}=K(i) \lambda_{i}$ then conditions (8) can be arranged as $\lambda_{0}>0, \ldots, \lambda_{T-1}>0$ and

$$
\left((\mathcal{A}+\mathcal{B K})_{T}-I\right)\left(\begin{array}{lll}
\lambda_{1}^{T} \lambda_{2}^{T} & \ldots & \lambda_{T-1}^{T} \lambda_{0}
\end{array}\right)^{T}<0,
$$

where $I$ stands for the identity matrix of appropriate size and $(\mathcal{A}+\mathcal{B K})_{T}$ is defined to be

$$
(\mathcal{A}+\mathcal{B K})_{T}:=\left(\begin{array}{cc}
0 & \mathcal{F}(0) \\
\operatorname{diag}(\mathcal{F}(1), \ldots, \mathcal{F}(T-1)) & 0
\end{array}\right)
$$

with $\mathcal{F}(i)=A(i)+B(i) K(i)$. As the modes are nonnegative so that $(\mathcal{A}+\mathcal{B K})_{T} \geq 0$ and thus we can apply Lemma 2.4 to conclude that $(\mathcal{A}+\mathcal{B K})_{T}$ is Schur. Hence, due to Lemma 2.1 one can conclude that the closed-loop system resulting from a periodic gain of the form $K(t+N T)=Y_{t} \operatorname{diag}\left(\lambda_{t}\right)^{-1}$, is asymptotically stable.

The necessity of conditions (7) and (8) can be shown using the same arguments as above.

The following corollary shows how one can design stabilizing positive gains, or equivalently, stabilizing nonnegative control signals.

Corollary 3.2: There exists a stabilizing nonnegative feedback law for system (6) with a periodic gain $K(t+T)=K(t)$ such that the closed-loop system is positive if and only if there exist $Y_{0}, \ldots, Y_{T-1} \in \mathbb{R}^{p \times n}$ and $\lambda_{0}, \ldots, \lambda_{T-1} \in \mathbb{R}^{n}$ satisfying conditions (7), (8) and the additional condition: $Y_{0} \geq 0, \ldots, Y_{T-1} \geq 0$.

If so, the closed-loop system is positive and asymptotically stable under the nonnegative control law $u(t)=K(t) x(t)$ with

$K(t+N T)=Y_{t} \operatorname{diag}\left(\lambda_{t}\right)^{-1}$, for $t \in\{0, \ldots, T-1\}, N \in \mathbb{Z}_{+}$. 


\section{State AND CONTROL CONSTRAints}

In general, the problem of designing stabilizing statefeedback controllers with structured or bounded gains is an NP-hard problem to solve [13]. In contrast, this problem can be efficiently solved for positive LTI systems and in particular for positive periodic systems.

In what follows, we consider the stabilization problem for system (6) with constrained states and control signals. In practice, we have to respect some prescribed upper and lower bounds:

$$
0 \leq x(t) \leq x_{\max }, u_{\min } \leq u(t) \leq u_{\max }, \forall t \in \mathbb{Z}_{+}
$$

such that $\left(x_{\max }, u_{\min }, u_{\max }\right) \in \mathbb{R}^{n} \times \mathbb{R}^{p} \times \mathbb{R}^{p}$ and $x_{\max }>0$, $u_{\min } \leq 0$ and $u_{\max } \geq 0$.

For this purpose, we define the set of desirable constraints as $\mathcal{C}\left(x_{\max }, u_{\min }, u_{\max }\right):=$

$$
\left\{(x, u) \in \mathbb{R}^{n} \times \mathbb{R}^{p} \mid 0 \leq x \leq x_{\max }, u_{\min } \leq u \leq u_{\max }\right\} .
$$

The domain of attraction of nonnegative initial conditions $x(0)=x_{0}$ associated to a given stabilizing control law $u(t)$ is defined to be $\mathbb{D}(u):=$

$$
\begin{gathered}
\left\{x_{0} \in \mathbb{R}_{+}^{n} \mid(x(t+N T), u(t+N T)) \in \mathcal{C}\left(x_{\max }^{t}, u_{\min }^{t}, u_{\max }^{t}\right),\right. \\
\left.\forall t \in\{0, \ldots, T-1\}, \forall N \in \mathbb{Z}_{+}\right\} .
\end{gathered}
$$

Also, we define a set $\mathcal{S}$ to be an inner approximation of the domain of attraction $\mathbb{D}(u)$ if $\mathcal{S}$ is a subset: $\mathcal{S} \subset \mathbb{D}(u)$. For the control design, we shall use a specific family of inner approximation sets formed by boxes.

Next, we address the problem of designing a stabilizing state-feedback law with an associated inner approximation for its domain of attraction. We first, treat the case when the states and the inputs are constrained to be nonnegative and bounded. The following result shows how to handle this task.

Theorem 4.1: Let $x_{\text {max }}^{t}, u_{\text {min }}^{t}(=0), u_{\text {max }}^{t}, t \in\{0, \ldots, T-$ $1\}$ be periodically prescribed bounds. Then for a stabilizing nonnegative state-feedback law $u(t)=K(t) x(t)$ such that the closed-loop system is positive, one can determine an inner approximation box $\mathbb{B}\left(\lambda_{0}\right) \subset \mathbb{D}(u)$ if and only if there exist $Y_{0}, \ldots, Y_{T-1} \in \mathbb{R}^{p \times n}$ and $\lambda_{0}, \ldots, \lambda_{T-1} \in \mathbb{R}^{n}$ satisfying conditions (7), (8) and the following inequalities

$$
Y_{t} \geq 0, Y_{t} \mathbb{1}_{n} \leq u_{\max }^{t}, \lambda_{t} \leq x_{\max }^{t}, t=0,1, \ldots, T-1
$$

If so, a nonnegative control law $u(t)=K(t) x(t)$ associated to the inner approximation box $\mathbb{B}\left(\lambda_{0}\right) \subset \mathbb{D}(u)$ can be determined as

$K(t+N T)=Y_{t} \operatorname{diag}\left(\lambda_{t}\right)^{-1}$ for $t \in\{0, \ldots, T-1\}, N \in \mathbb{Z}_{+}$.

Proof: Sufficiency: Assume that inequalities (7)-(8)(10) are feasible and select any solution $Y_{0}, \ldots, Y_{T-1}$ and $\lambda_{0}, \ldots, \lambda_{T-1}$. Define $u(t+N T)=Y_{t} \operatorname{diag}\left(\lambda_{t}\right)^{-1} x(t+$ $N T)$ for $t \in\{0, \ldots, T-1\}$. With regards to previous results, it has been shown that conditions (7) and (8) are equivalent to the modes positivity of the closed-loop system and its stability. Also, as $Y_{t} \geq 0$ then $K(t+N T)=Y_{t} \operatorname{diag}\left(\lambda_{t}\right)^{-1} \geq 0$ and consequently the nonnegativity of the feedback control is guaranteed $u(t)=K(t) x(t) \geq 0$.
It remains to show that for $\lambda_{0}$ the box $\mathbb{B}\left(\lambda_{0}\right)$ represents an inner approximation of the domain of attraction $\mathbb{D}(u)$. For this, note that condition (8) is equivalent to

$$
\left\{\begin{array}{l}
(A(0)+B(0) K(0)) \lambda_{0}<\lambda_{1} \\
(A(1)+B(1) K(1))(A(0)+B(0) K(0)) \lambda_{0}<\lambda_{2} \\
\vdots \\
(A(T-1)+B(T-1) K(T-1)) \ldots(A(0)+B(0) K(0)) \lambda_{0}<\lambda_{T-1}
\end{array}\right.
$$

then as a result of Theorem 2.7 we can conclude that the closed-loop system is periodically invariant for the collection formed by the boxes $\mathbb{B}\left(\lambda_{0}\right) \ldots \mathbb{B}\left(\lambda_{T-1}\right)$. That is whenever $x(0)<\lambda_{0}$ we have that $x(t+N T)<\lambda_{t}, t=0, \ldots, T-1$, $N \in \mathbb{Z}_{+}$. Thus, it holds that $x(t+N T)<\lambda_{t} \leq x_{\max }^{t}$ and also it follows

$$
\begin{aligned}
u(t+N T) & =K(t+N T) x(t+N T) \\
& \leq K(t+N T) \lambda_{t}=Y_{t} \mathbb{1}_{n} \leq u_{\max }^{t} .
\end{aligned}
$$

Necessity: Let $u(t)=K(t) x(t) \geq 0$ be any stabilizing nonnegative control $(K(t) \geq 0)$ such that the closed-loop system is positive. Then by Theorem 2.8 the closed-loop system has a periodic invariant collection of open boxes $\mathbb{B}\left(\gamma_{0}\right) \ldots \mathbb{B}\left(\gamma_{T-1}\right)$ associated to $\gamma_{0}>0, \ldots, \gamma_{T-1}>0$. Note that for any scalar $\alpha>0$ it holds that $\mathbb{B}\left(\alpha \gamma_{0}\right) \ldots \mathbb{B}\left(\alpha \gamma_{T-1}\right)$ is also a periodic invariant collection for the closed-loop system. Since there exists always $\alpha>0$ sufficiently small such that $\alpha \gamma_{t} \leq x_{\max }^{t}$ and $\alpha K(t) \gamma_{t} \leq u_{\max }^{t}$, for $t=1, \ldots, T-1$ one can define with such $\alpha: \lambda_{0}:=\alpha \gamma_{0}, \ldots, \lambda_{T-1}:=\alpha \gamma_{T-1}$ and $Y_{0}:=$ $K(0) \operatorname{diag}\left(\lambda_{0}\right), \ldots, Y(T-1):=K(T-1) \operatorname{diag}\left(\lambda_{T-1}\right)$. Thus, by construction and by making use of the fact that $\mathbb{B}\left(\lambda_{0}\right) \ldots \mathbb{B}\left(\lambda_{T-1}\right)$ is also a periodic invariant collection for the closed-loop system, we can see that for any $x(0) \in \mathbb{B}\left(\lambda_{0}\right)$ it holds $x(t)<\lambda_{t} \leq x_{\max }^{t}$. Since $K(t) \geq 0$ we also have for $t=0, \ldots, T-1, N \in \mathbb{Z}_{+}$

$$
0 \leq u(t)=K(t) x(t) \leq K(t) \lambda_{t}=Y_{t} \mathbb{1}_{n} \leq u_{\max }^{t} .
$$

Consequently, we have got an inner approximation box $\mathbb{B}\left(\lambda_{0}\right) \subset \mathbb{D}(u)$ and we have seen that it holds $Y_{t} \geq 0, Y_{t} \mathbb{1}_{n} \leq$ $u_{\text {max }}^{t}, \lambda_{t} \leq x_{\text {max }}^{t}, t=0,1, \ldots, T-1$. In addition, due to modes positivity and stability consideration, one can deduce that conditions (7), (8) are also satisfied.

Now, we present a numerical design for stabilizing controls with non symmetrical lower and upper bounds.

Corollary 4.2: Let $x_{\max }^{t}, u_{\min }^{t}, u_{\max }^{t}, t \in\{0, \ldots, T-1\}$ be periodically desirable bounds. Then for a stabilizing statefeedback law $u(t)=K(t) x(t)$ such that the closed-loop system is positive, one can determine an inner approximation box $\mathbb{B}\left(\lambda_{0}\right) \subset \mathbb{D}(u)$ if and only if there exist $Y_{0}, \ldots, Y_{T-1} \in \mathbb{R}^{p \times n}$, $Z_{0}, \ldots, Z_{T-1} \in \mathbb{R}^{p \times n}$ and $\lambda_{0}, \ldots, \lambda_{T-1} \in \mathbb{R}^{n}$ such that

$$
\begin{aligned}
& A(i) \operatorname{diag}\left(\lambda_{i}\right)+B(i)\left(Y_{i}-Z_{i}\right) \geq 0, \quad 0 \leq i \leq T-1(12) \\
& \left\{\begin{array}{l}
\lambda_{0}>0, \lambda_{1}>0, \ldots, \lambda_{T-1}>0 \\
A(0) \lambda_{0}+B(0)\left(Y_{0}-Z_{0}\right) \mathbb{1}_{n}<\lambda_{1} \\
A(1) \lambda_{1}+B(1)\left(Y_{1}-Z_{1}\right) \mathbb{1}_{n}<\lambda_{2} \\
\vdots \\
A(T-2) \lambda_{T-2}+B(T-2)\left(Y_{T-2}-Z_{T-2}\right) \mathbb{1}_{n}<\lambda_{T-1} \\
A(T-1) \lambda_{T-1}+B(T-1)\left(Y_{T-1}-Z_{T-1}\right) \mathbb{1}_{n}<\lambda_{0}
\end{array}\right.
\end{aligned}
$$




$$
\left\{\begin{array}{l}
Y_{t} \geq 0, Z_{t} \geq 0, \\
Y_{t} \mathbb{1}_{n} \leq u_{\max }^{t}, Z_{t} \mathbb{1}_{n} \leq-u_{\min }^{t}, \lambda_{t} \leq x_{\max }^{t},
\end{array}\right.
$$

for $t=0,1, \ldots, T-1$. If so, a stabilizing control law $u(t)=$ $K(t) x(t)$ associated to the inner approximation box $\mathbb{B}\left(\lambda_{0}\right):=$ $\left\{x \in \mathbb{R}_{+}^{n} \mid x<\lambda_{0}\right\} \subset \mathbb{D}(u)$ can be characterized as $K(t+$ $N T)=\left(Y_{t}-Z_{t}\right) \operatorname{diag}\left(\lambda_{t}\right)^{-1}$, for $t \in\{0, \ldots, T-1\}, N \in$ $\mathbb{Z}_{+}$.

Proof: The proof is similar to the one of Theorem 4.1. It suffices to take into account the following facts. For the sufficiency part one can use the gains form $K(t)=\left(Y_{t}-\right.$ $\left.Z_{t}\right) \operatorname{diag}\left(\lambda_{t}\right)^{-1}$ for $t \in\{0, \ldots, T-1\}$ by considering the following inequalities $x(t+N T)<\lambda_{t} \leq x_{\max }^{t}$. Since $u(t)=$ $K(t+N T) x(t+N T)=\left(Y_{t}-Z_{t}\right) \operatorname{diag}\left(\lambda_{t}\right)^{-1} x(t+N T)$ with $Y_{t} \geq 0, Z_{t} \geq 0, \lambda_{t}>0$ and $x(t+N T) \geq 0$, we can deduce that $u(t)=K(t+N T) x(t+N T) \geq-Z_{t} \operatorname{diag}\left(\lambda_{t}\right)^{-1} x(t+$ $N T)$. As $x(t+N T)<\lambda_{t}$ and $Z_{t} \geq 0$ we have that

$u(t)=K(t+N T) x(t+N T) \geq-Z_{t} \operatorname{diag}\left(\lambda_{t}\right)^{-1} \lambda_{t}=-Z_{t} \mathbb{1}_{n}$

In summary, by using this kind of argument, we can establish the bounds $u_{\min }^{t} \leq-Z_{t} \mathbb{1}_{n} \leq u(t)=K(t+N T) x(t+N T) \leq$ $Y_{t} \mathbb{1}_{n} \leq u_{\max }^{t}$.

Note that these inequalities cannot be strict since the matrices $Z_{t}$ or $Y_{t}$ may possess some line with zero entries.

Also, for the necessity part one can decompose the gain $K(t)$ into the difference of two positive matrices, its negative and positive parts, i.e., $K(t)=K^{+}(t)-K^{-}(t)$ such that $K^{+}(t) \geq 0$ and $K^{-}(t) \geq 0$. Thus, one can construct

$$
Y_{t}=K^{+}(t) \operatorname{diag}\left(\lambda_{t}\right) \geq 0, Z_{t}=K^{-}(t) \operatorname{diag}\left(\lambda_{t}\right) \geq 0,
$$

$t \in\{0, \ldots, T-1\}$, and follow the same line of argument previously used for Theorem 4.1.

\section{Enlarging the Domain of Attraction}

It is of great importance to design a large set of initial conditions that guarantee stability in the presence of states and/or control constraints. The task here is to address the issue of enlargement of the domain of attraction by computing a larger inner approximation of it. For this purpose we have seen that a natural inner estimate set of the domain of attraction consists of a simple box $\mathbb{B}(v):=\left\{x \in \mathbb{R}_{+}^{n} \mid x<v\right\}$. For this box it is possible to maximize easily different measures of its shape. For instance, this can be done by maximizing the length of its contours or equivalently by maximizing $p(\mathbb{B}(v))=\mathbb{1}_{n}^{T} v$.

In the case of nonnegative control one can compute such inner approximation based on the result of Theorem 4.1. Hence, this can be achieved by solving the following LP problem:

$$
\min -\mathbb{1}_{n}^{T} \lambda_{0} \quad \text { subject to: }(7),(8) \text { and (10) }
$$

Also, the other case with asymmetrical bounds on the control can be similarly treated based on Corollary 4.2 and can be treated by solving

$$
\min -\mathbb{1}_{n}^{T} \lambda_{0} \quad \text { subject to: }(12),(13) \text { and }(14) .
$$

Example 5.1: Consider system (6) described by the following modes

$$
\begin{aligned}
& A(0)=\left(\begin{array}{ll}
0.3178 & 0.1302 \\
0.5877 & 0.2544
\end{array}\right), B(0)=\left(\begin{array}{c}
-0.0063 \\
0.5245
\end{array}\right), \\
& A(1)=\left(\begin{array}{ll}
-0.7508 & 0.5173 \\
-0.5002 & 0.5592
\end{array}\right), B(1)=\left(\begin{array}{c}
0.3692 \\
0.1792
\end{array}\right) .
\end{aligned}
$$

Suppose that its states and control signals are constrained to respect the following prescribed bounds $x_{\max }^{0}=$ $\left(\begin{array}{ll}1 & 1\end{array}\right)^{T}, x_{\max }^{1}=\left(\begin{array}{ll}0.5 & 0.5\end{array}\right)^{T}, \quad\left(u_{\min }^{0}, u_{\max }^{0}\right)^{2}=$ $\left(\begin{array}{ll}-1 & 1\end{array}\right)^{T},\left(u_{\min }^{1}, u_{\max }^{1}\right)=\left(\begin{array}{ll}-0.5 & 2\end{array}\right)^{T}$; that is $0 \leq x(2 t) \leq$ $\left(\begin{array}{ll}1 & 1\end{array}\right)^{T},-1 \leq u(2 t) \leq 1$ and $0 \leq x(1+2 t) \leq$ $\left(\begin{array}{ll}0.5 & 0.5\end{array}\right)^{T},-0.5 \leq u(1+2 t) \leq 2$.

As a result, we have solved the LP problem (16) by using linprog Matlab function. We have obtained the following optimal solution $\lambda_{0}=\left(\begin{array}{ll}0.7112 & 1.0000\end{array}\right)^{T}$, $\lambda_{1}=\left(\begin{array}{ll}0.3583 & 0.5000\end{array}\right)^{T}, Y_{0}=\left(\begin{array}{ll}0.2605 & 0.2827\end{array}\right)$, $Y_{1}=\left(\begin{array}{ll}1.000 & 0.0000\end{array}\right), Z_{0}=\left(\begin{array}{ll}0.4994 & 0.3723\end{array}\right), Z_{1}=$ (0.0000 0.1954).

The gains $K(0)$ and $K(1)$ of the designed state-feedback control law are computed according to the established formula $K(t+N T)=\left(Y_{t}-Z_{t}\right) \operatorname{diag}\left(\lambda_{t}\right)^{-1}$ according to Corollary 4.2 and LP problem (16). We have got the following gains

$K(0)=(-0.3360 \quad-0.0896), K(1)=\left(\begin{array}{ll}2.7912-0.3907\end{array}\right)$.

Note that these gains ensure the positivity of each mode and leads to a quite good inner approximation $\mathbb{B}\left([0.71121]^{T}\right) \subset$ $\mathbb{D}(u) \subset \mathbb{B}\left(\left[\begin{array}{ll}1 & 1\end{array}\right]^{T}\right)$. Hence, the resulting state-feedback control stabilizes the closed-loop system, maintains it positive and fulfills the imposed state and control constraints for all initial conditions in the box $\mathbb{B}\left([0.71121 .0000]^{T}\right)$. These desired properties can be noticed from the evolution of the closedloop system and its control signals which are depicted in figure 1 for randomly generated initial conditions in the box $\mathbb{B}\left([0.71121 .0000]^{T}\right)$.

\section{CONCLuSions}

An efficient treatment has been proposed in order to address the problem of stabilizing a periodic discrete-time positive system and maintaining its positivity. Also, we have established a relationship between stability of a positive periodic system and its periodic invariance with respect to a collection of boxes. Based on this connection, we have demonstrated how one can tackle the synthesis problem with respect to state and control constraints.

\section{ACKNOWLEDGEMENT}

The authors would like to thank the reviewers for their valuable comments and suggestions that led to a much improved manuscript. This work was supported by Portuguese funds through the CIDMA - Center for Research and Development in Mathematics and Applications, and the Portuguese Foundation for Science and Technology ("FCT-Fundação para a Ciência e a Tecnologia"), within project PEst-OE/MAT/UI4106/2014. 


\section{REFERENNCIAS}

[1] M. Ait Rami. Solvability of static output-feedback stabilization of positive LTI systems. Systems \& control letters, 60: 704-708, 2011.

[2] M. Ait Rami, D. Napp. Characterization and Stability of Autonomous Positive Descriptor Systems. IEEE Trans. Automat. Control, 57(10): 2668-2673, 2012.

[3] M. Ait Rami, F. Tadeo, U. Helmke. Positive observers for linear positive systems, and their implications. International Journal of Control, 84:416 - 725, 2011.

[4] M. Ait Rami. Stability and stabilization of positive systems with time varying delays. Lecture notes in control and information sciences, 389: 205-215, Springer, 2009.

[5] M. Ait Rami and F. Tadeo. Controller Synthesis for positive Linear Systems with Bounded controls. IEEE Trans. Syst. And Circuits, 54: 151-155, 2007.

[6] S. Bittanti, P. Colaneri, G. De Nicolao. An algebraic Riccati equation for the discrete-time periodic prediction problem. Systems \& Control Letters, 14 (1): 71-78, 1990.

[7] S. Bittanti, P. Colaneri, G. De Nicolao. The Periodic Riccati Equation. in: The Riccati Equation S. Bittanti, A.J. Laub and J.C. Willems, Eds. Berlin: Springer Verlag, 127-162, 1991.

[8] S. Bittanti. Deterministic and stochastic linear periodic systems. In Time series and linear systems, volume 86 of Lecture Notes in Control and Inform. Sci., p. 141-182. Springer, Berlin, 1986.

[9] S. Bittanti and P. Colaneri. Invariant representations of discretetime periodic systems. Automatica, 36(12): 1777-1793, 2000.

[10] F. Blanchini and W. Ukovich. Linear programming approach to the control of discrete-time periodic systems with uncertain inputs. $J$. of Optimization theory and Applications, 78(3): 523-539, 1993.

[11] N. Bougatef, M. Chaabane, O. Bachelier, and D. Mehdi. Stability and stabilization of constrained positive discrete-time periodic systems. 8th International Multi-Conference on Systems, Signals and Devices (SSD), 2011.

[12] N. Bougatef, D. Mehdi, O. Bachelier, and M. Chaabane. Constrained control of positive discrete-time periodic systems with delays. 2011 50th IEEE Conference on Decision and Control and European Control Conference, 2011.

[13] V. Blondel and J. N. Tsitsiklis. NP-hardness of some linear control design problems. SIAM J. Control Optim., 35(6): 2118-2127, 1997.

[14] S. Bittanti, and P. Colaneri. Periodic Systems: Filtering and Control. Springer-Verlag 2009.

[15] P. Bolzern, and P. Colaneri. The Periodic Lyapunov Equation. SIAM J. on Matrix Analysis and Applications, 9(4): 499-512, 1988.

[16] C. Briat. Robust stability analysis of uncertain linear positive systems via integral linear constraints: $L_{1}$-gain and $L_{\infty}$-gain characterizations. International Journal of Robust and Nonlinear Control, 23: 1932-1954, 2013.

[17] R. Bru and V. Hernández. Structural properties of discrete-time linear positive periodic systems. Linear Algebra Appl., 121: 171183, 1989.

[18] R. Bru, C. Coll, V. Estruch and E. Sánchez. Output feedback of forward-backward periodic systems stability. IEEE Trans. Automat. Control, 45(2): 319-323, 2000.

[19] R. Bru, R. Cantó, B. Ricarte. Modelling nitrogen dynamics in citrus tree, Mathematical and Computer Modelling,38:975-987, 2003.

[20] R. Bru, S. Romero and E. Sánchez. Structural properties of positive periodic discrete-time linear systems: canonical forms. Appl. Math. Comput., 153(3): 697-719, 2004.

[21] R. Bru, S. Romero, E. Sánchez. Reachability indices of periodic positive systems via positive shift-similarity Linear Algebra and Its Applications, vol. 429 (5), 1288-1301, 2008

[22] R. Cantó, B. Ricarte, A. M. Urbano. A dynamic nitrogen uptake and mobilization model for young evergreen trees. Mathematical and Computer Modelling, 50(5-6): 939-946, 2009.

[23] B. Cantó, C. Coll, E. Sánchez. Positive N-periodic descriptor control systems. Systems \& Control Letters 53(5): 407-414, 2004.

[24] B. Cantó, C. Coll, E. Sánchez. On positive behaviour of periodic control systems. Applied Mathematics and Computation, 161(3):779-786, 2005.

[25] C. E. De Souza, A. Trofino. An LMI Appraoch to Stabilization of Linear Discrete-Time Periodic Systems. Int. J. of Control, Vol. 73 (8): 696-703, 2000.

[26] O. M. Grasselli, S. Longhi, A. Tornambe, P. Valigi. Robust Output Regulation and Tracking for Linear Periodic Systems under Structured Uncertainties. Automatica, 32(7): 1015-1019, 1996.
[27] L. Farina and S. Rinaldi. Positive linear systems: Theory and applications. Wiley-Interscience, New York, 2000

[28] D. S. Flamm. A new shift-invariant representation for periodic linear systems Systems \& Control Letters, 17(1): 9-14, 1991.

[29] G. Floquet. Sur les équations différentielles linéaires á coefficients périodiques. Annales scientifiques de l'Ecole Normale Supérieure, 2(12): 47-88, 1883.

[30] W. M. Haddad, V. Chellaboina, and Q. Hui. Nonnegative and Compartmental Dynamical Systems. Princeton, NJ: Princeton University Press, 2010.

[31] U. Helmke, E. I. Verriest. Structure and parametrization of periodic linear systems Math. Control Signals Syst., 23: 67-99, 2011.

[32] A. Lyapunov. Sur une serié relative á la théorie des équations différentielles linéaires á coefficients périodiques. Comptes Rendus de Acad. des Sciences, 123(26): 1248-1252, 1896.

[33] T. Kaczorek. Positive 1D and 2D Systems Springer Verlag, 2001.

[34] C. Y. Kao, A. Megretski, U. T. Jonsson. A Cutting Plane Algorithm for Robustness Analysis of Periodically Time-Varying Systems. IEEE Transactions on Automatic Control, 46 (4), 2001.

[35] A. Rantzer. Distributed Control of Positive Systems. IEEE CDC conf. and ECC conf., Orlando, 2011.

[36] R. Bru, C. Coll, V. Hernández and E. Sánchez. Geometrical conditions for the reachability and realizability of positive periodic discrete systems Linear Algebra Appl., 256: 109-124, 1997.

[37] P. Van Dooren, and J. Sreedhar. When is a periodic discrete-time system equivalent to a time-invariant one? Linear Algebra and its Applications, 212/213: 131-151, 1994.

[38] E. I. Verriest. Alternating discrete time systems: invariants, parametrization and realization. In: Proceedings of conference on information sciences and systems, Princeton, pp 952-957, 1988.

[39] V. Yakubovich, A. Fradkov, D. Hill, and A. Proskurnikov. Dissipativity of T-periodic linear systems. IEEE Transactions on Automatic Control, 52(6): 1039-1047, 2007.

[40] B. Zhou, G. R. Duan, Z. Lin. Parametric periodic Lyapunov equation with application in semi-global stabilization of discretetime periodic systems subject to actuator saturation. Automatica, 47 (2): 316-325, 2011.

[41] B. Zhou, G. R. Duan, Z. Lin. On Semiglobal Stabilization of Discrete-Time Periodic Systems With Bounded Controls. IEEE Tran. on Circuits and Systems II-express Briefs, 58 (7):452-456, 2011. 

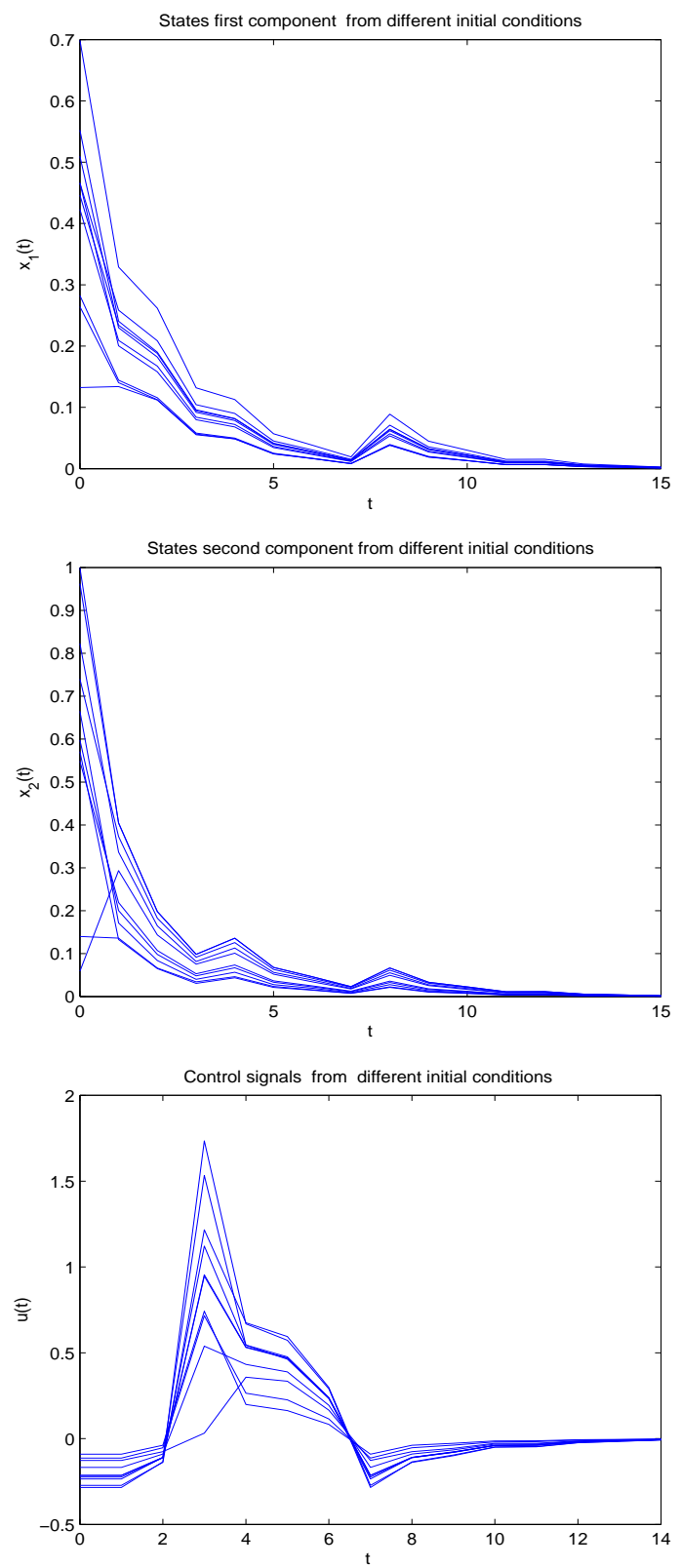

Figura 1. Evolution of the states and control signals from randomly generated initial conditions in the box $\mathbb{B}\left(\left[\begin{array}{lll}0.7112 & 1.0000\end{array}\right]^{T}\right)$. 